\title{
A MODIFIED METHOD FOR MODELLING OF SPREAD FOOTING UNDER UNIFORM DISTRIBUTED LOAD USING WINKLER'S MODEL
}

\author{
Peyman Mianji*, Ehsan Seyedi Hosseininia \\ Department of Civil Engineering, Ferdowsi University of Mashhad, Azadi Sq., \\ Mashhad, Iran
}

[Received 09 October 2017. Accepted 18 February 2019]

doi: 10.7546/JTAM.49.19.01.04

\begin{abstract}
Recent developments and advancements in soil models and computer programs failed to prevent structural engineering to use one the most elementary theories in modeling of the soil-structure interaction, i.e. Winkler's model. Superseding the soil with all its complexity with a series of uncoupled, independent springs whose stiffness known as the modulus of subgrade reaction $\left(K_{s}\right)$, imposes many non-avoidable errors to the model. In the first two parts of the paper, the impact of soil-foundation system stiffness on the $K_{s}$ distribution alongside the precision of the theoretical relations for $K_{s}$ estimation are investigated as the basis for the proposed dividing technique presented in the rest of the article.
\end{abstract}

KEY WORDS: Winkler's model; modulus of subgrade reaction; soil-foundation system rigidity; experimental and theoretical relation of $K_{s}$ estimation; dividing technique.

\section{INTRODUCTION}

One of the common interfaces between the structural and geotechnical engineering is a proper modeling of the soil-structure interaction that can provide both the simplicity and precision. No proper modeling leads to a conservative design either for the structure and foundation or system inefficiency and damage. The model of beam on elastic soil, can model the soil-structure interface as an infinite, closely spaced springs, which originally is proposed for railway lines by Winkler [1], and is widely used for modeling of the soil-foundation interface. In this context, defining a suitable value for the spring stiffness $\left(K_{s}\right)$ is a challenging subject while a wide range of methods have been introduced over the decades by several researchers as classified below: 1 - Plate load test (PLT afterwards); 2 - Consolidation test; 3 - Triaxial test; 4 - CBR test; and 5 - Empirical and theoretical relations [2].

\footnotetext{
${ }^{*}$ Corresponding author e-mail: peyman.mianji@gmail.com
} 
Among the methods, empirical and theoretical relations as well as the PLT are mostly utilized in estimating the modulus of subgrade reaction $\left(K_{s}\right)$. However, the $K_{s}$ derived from the PLT should be considered with some correction factors, as the rigidity of the plate and the actual foundation is not the same; the former is always rigid but the latter could be rigid or with different degrees of rigidity [3]. Additionally, much effort has been devoted for empirical and theoretical relations; Biot [4], Vesic [5], and the equation based on the theory of elasticity [6] are the most commonly used relations compare to others. Sadrekarimi and Akbarzad [2], were appraised the suitability and accuracy of the aforementioned relations using 2D finite element software, and showed that the Vesic relation has a better agreement with the soft soil model for Tabriz Marl.

Inherently, the Winkler's model cannot transmit any shear stress owing to the lack of spring coupling, while assigning a single value of $K_{s}$ for whole the foundation resulted a uniform settlement and no bending moments, which makes the model's error twofold. Filonenko-Borodich, Hetenyi, and Pasternak [3] have taken part in the modification of the Winkler's model by adding tension membrane or plate to be able to incorporate the shear behavior into their models. In the same way, Vlasov and Leot'ev [7] introduced a new parameter $(\gamma)$ to heed the shear strain within the soil continuum. Later, the Modified Vlasov Model was introduced by Vallabhan and Das [8] with a new calculation method for the parameter $\gamma$. Daloglu and Vallabhan [9] investigated the Biot [4], Vesic [5] and Vlasov and Leot'ev [7] models through a comparative study with the Winkler's equation. For concentrated load the Vlasov model is in well agreement with the Winkler's model, whereas for distributed load the Vlasov model gives conservative results for both settlement and bending moment.

According to the literatures, the $K_{s}$ value is dependent on the foundation's dimension, soil condition, load level, super structure, soil-foundation system rigidity and the soil model $[3,10]$ which causes no unique value to be found even for a certain specified condition. Ziaie-Moayed and Janbaz [10] studied the effect of different parameters, i.e. size effect, shape effect, embedment depth, and rigidity on the distribution of the $K_{s}$ beneath the foundation in clayey soils using a 3D finite element software. Arnold et al. [11] conducted a series of centrifuge tests on both sand and clay in order to find the impact of soil type, strength, stiffness and layering the soil, foundation stiffness, and load magnitude on the stress distribution. Furthermore, similar investigations have been fulfilled by Farouk and Farouk [3,12], where the contact stress distribution as well as the $K_{s}$ distribution of a beam with different rigidities were obtained.

Despite all the attempts have been made in amendment of the Winkler's model through developing new models, there is still a great deal of interest among practical engineers and software makers in using this theory. As a result, a variety of finite 
element software are available for ordinary calculation purpose based on modulus of subgrade reaction model. On the other hand, the complexity involved in estimation of soil parameters properly has limited the use of more advanced soil models and programs.

In this paper, the impact of soil-foundation stiffness on the $K_{s}$ distribution of square foundations under a uniform distributed load is persuaded. In the following, the Biot, Vesic and elastic-based relations of subgrade modulus estimation are examined by means of 2D and 3D finite element codes using elastic perfectly-plastic model for soil, to find the most accurate one. Ultimately, a new technique of assigning the $K_{s}$ is provided and evaluated by comparing the results of a 3D finite element software with that of a software based on Winkler's theory.

\section{Material and Model Specifications}

PLAXIS 2D (V. 8.2), PLAXIS 3D FOUNDATION (V. 1.1), and SAFE (V. 8.1) are the softwares used in different stages of investigation, respectively. A homogeneous sand soil with the enumerated properties given in Table 1, was modeled by linear elastic model in both finite element software and SAFE software. Moreover, the foundation was considered as an elastic material with the properties provided in Table 2. In the numerical models, the soil-foundation interface was described as rigid condition. In terms of boundary condition, full fixity and roller fixity were opted for horizontal (bottom) and vertical sides, respectively. Moreover, through several numerical modeling, it was found out that the analysis in a medium with dimensions seven times of footing dimensions in all directions, does not affect by the boundary conditions.

Table 1. Soil properties

\begin{tabular}{lclc}
\hline \hline PARAMETER & VALUE & PARAMETER & VALUE \\
\hline Material type & Drained & Young's modulus $\left(E_{s}\right)\left(\mathrm{kN} / \mathrm{m}^{2}\right)$ & $32.3 \times 10^{3}$ \\
Dry unit weight $\left(\gamma_{d}\right)\left(\mathrm{kN} / \mathrm{m}^{3}\right)$ & 17 & Poission's ratio $(\mu)$ & 0.3 \\
\hline \hline
\end{tabular}

Table 2. Concrete properties

\begin{tabular}{lcccc}
\hline \hline PARAMETER & $\begin{array}{c}\text { Material } \\
\text { type }\end{array}$ & $\begin{array}{c}\text { Concrete unit weight } \\
\gamma_{c}\left[\mathrm{kN} / \mathrm{m}^{3}\right]\end{array}$ & $\begin{array}{c}\text { Poisson ratio } \\
\mu\end{array}$ & $\begin{array}{c}\text { Young's modulus } \\
\left.E_{c}\right)\left[\mathrm{kN} / \mathrm{m}^{2}\right]\end{array}$ \\
\hline VALUE & Non-porous & 24 & 0.15 & $21.8 \times 10^{6}$ \\
\hline \hline
\end{tabular}




\section{The Impact of System Rigidity on $K_{s}$ Distribution}

As it mentioned in the introduction section, the soil-foundation rigidity has a direct effect on the $K_{s}$ distribution. In this part, $10 \times 10$ square footings with different thicknesses of $0.1,0.5,1,2$, and 4 meters subjected to a uniform distributed load of $100 \mathrm{kPa}$ were modeled by PLAXIS 2D and PLAXIS 3D foundation software to study the impact of system rigidity on $K_{s}$ distribution.

In this research the rigidity of the system is defined using DIN Standard (2005), through the Equation (1) [12]

$$
K=\left(\frac{d_{f}}{L}\right)^{3}\left(\frac{E_{c}}{12 E_{s}}\right),
$$

wherein, $d_{f}$ is the foundation thickness, $L$ is the length of foundation, and $E_{c}$ and $E_{s}$ are the foundation and soil Young's modulus, respectively. Arnold et al. [11] classified the foundation's rigidity into four groups: 1 - Absolutely flexible: the value of $K$ equals to zero; 2 - Semi-flexible: values around $0.01 ; 3$ - Semi-rigid: $K$ around 0.1 ; and 4 - Rigid: $K$ more than 0.1 to infinity.

Different thicknesses represent the different rigidities; the value of $K$ and corresponding category, according to the Arnold et al. [11] classification, is provided in Table 3.

Table 3. $K$ values for foundations with different thickness and corresponding category

\begin{tabular}{cccc}
\hline \hline THICKNESS $[\mathrm{m}]$ & $K$ VALUE & RANGE & CATEGORY \\
\hline 0.1 & 0.000055 & 0 & Flexible \\
0.5 & 0.007 & $0-0.01$ & Semi-flexible \\
1 & 0.055 & $0.01-0.1$ & Semi-rigid \\
2 & 0.431 & $0.1-\infty$ & Rigid \\
4 & 3.5 & $0.1-\infty$ & Rigid \\
\hline \hline
\end{tabular}

The normalized $K_{s}$ distribution of the foundations with different rigidities are presented in Fig. 1 and Fig. 2, which was obtained from PLAXIS 3D and 2D software, respectively. For this purpose, 10 points in every 0.5 meters is defined at the middle line of the footing (from center to side) to record the contact stresses and settlements. For normalization, the $K_{s}$ obtained in each point divided by the $K_{s}$ of the center of footing. The number 0 in $x$-axis, represent the center of footing, while only half of footing is depicted.

It can be seen from Figs. 1 and 2 that with increasing the rigidity, $K_{s}$ increases at a small distance from the side of foundation, while it is invariant at the center. In the case of flexible foundation, the $K_{s}$ is almost constant. Moreover, the $K_{s}$ distribution of semi-rigid and two rigid cases are entirely identical. 


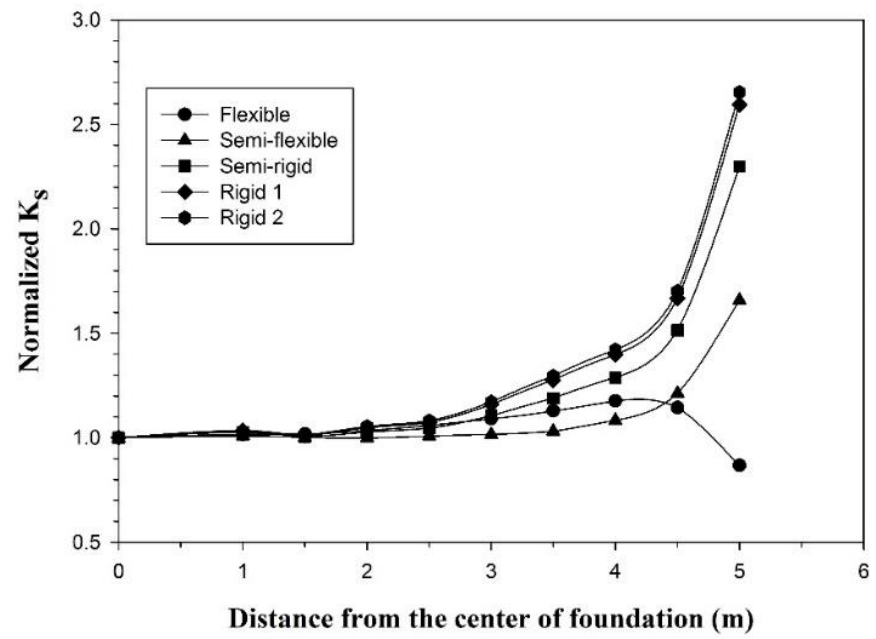

Fig. 1. Normalized $K_{s}$ distribution of $10 \times 10$ footings with distinct rigidities (PLAXIS 3D) according to Table 3 .

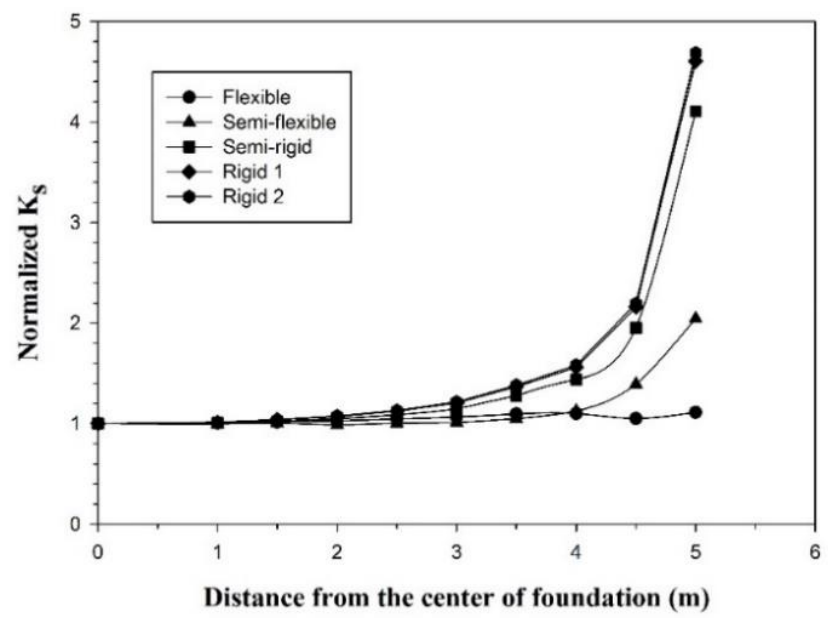

Fig. 2. Normalized $K_{s}$ distribution of $10 \times 10$ footings with distinct rigidities (PLAXIS 2D) according to Table 3 .

\section{The Exactitude of the $K_{s}$ Estimation Relations}

The $K_{s}$ computed from the Biot and Vesic relations as well as the relation derived from the theory of elasticity were compared with that of obtained from 3D finite element analysis, for foundations with dimensions: $10 \times 10,20 \times 20,30 \times 30,40 \times 40$, and $50 \times 50$. 


$$
\begin{aligned}
K_{s} & =\frac{0.95 E_{s}}{B\left(1-\mu^{2}\right)}\left[\frac{B^{4} E_{s}}{\left(1-\mu^{2}\right) E_{f} I}\right]^{0.108} & & \text { Biot } \\
K_{s} & =\frac{0.65 E_{s}}{B\left(1-\mu^{2}\right)} \sqrt[12]{\frac{E_{s} B^{4}}{E_{f} I}} & & \text { Vesic } \\
K_{s} & =\frac{E_{s}}{B\left(1-\mu^{2}\right) m I_{s} I_{f}} & & \text { Based on elastic theory }
\end{aligned}
$$

where $E_{s}=$ Young's modulus of the soil, $\mu=$ Poisson's ratio, $B=$ the breadth of footing, $I=$ moment of inertia, $E_{f}=$ footing Young's modulus, $m=$ number of corners contributing to settlement, 4,2 , and 1 for center, side and edge of foundation respectively, and $I_{s}$ and $I_{f}=$ influence factors which depend on shape of footing, aspect ratio, and embedment depth.

The $K_{s}$ values of foundations using Eqs. 2, 3, and 4, and that of procured from 3D finite element analysis is presented in Table 4 . It is noted that the $K_{s}$ quantities of elastic-based theory and PLAXIS 3D methods, provided in Table 4, are the average of center, side and corner for the former method and center and side for the latter one. The reason for not using the corner values in PLAXIS 3D method is the unrealistic large values at edges that resulted from using the linear elastic model for soil. In all cases, the footings' thicknesses were selected in such a way to behave as a semi-rigid and do not have any influence on the $K_{s}$ values.

The SAFE software is one of the softwares that use the Winkler's theory in modeling of the soil. It was utilized in this part of study to verify the $K_{s}$ relations. Accordingly, the $10 \times 10$ footings were modeled by SAFE for the $K_{s}$ provided in Table 4 and various rigidities. The percentages of discrepancies are listed in Table 5. The positive percentage shows that the settlement calculated by SAFE is less than the value obtained from PLAXIS 3D Foundation and the negative percentage vice versa.

According to the Table 4 and Table 5, the $K_{s}$ appraised by the relation based on theory of elasticity and Biot have a better agreement with the finite element results. While the Vesic relation does not show an acceptable consistency.

Table 4. $K_{s}$ estimation using theoretical relations and 3D finite element software

\begin{tabular}{ccccc}
\hline \hline $\begin{array}{c}\text { FOUNDATIONS } \\
\text { DIMENTION }\end{array}$ & BIOT & VESIC & $\begin{array}{c}K_{s}\left[\mathrm{kN} / \mathrm{m}^{2}\right] \\
\text { ELASTIC THEORY }\end{array}$ & PLAXIS 3D \\
\hline $10 \times 10$ & 4792 & 3021 & 4100 & 4486 \\
$20 \times 20$ & 2396 & 1511 & 2050 & 2433 \\
$30 \times 30$ & 1579 & 1007 & 1366 & 1634 \\
$40 \times 40$ & 1198 & 755 & 1025 & 1200 \\
$50 \times 50$ & 958 & 604 & 820 & 991 \\
\hline \hline
\end{tabular}


Table 5. The settlement difference between the PLAXIS 3D and SAFE software using various $K_{s}$

\begin{tabular}{lccc}
\hline \hline $10 \times 10$ FOOTING & BIOT & VESIC & ELASTIC THEORY \\
\hline Flexible & +24.7 & -19.3 & +12.1 \\
Semi-flexible & +17.6 & -30.6 & +3.9 \\
Semi-rigid & +5.8 & -49.2 & -9.9 \\
Rigid 1 & +1.5 & -56.2 & -15.1 \\
Rigid 2 & +0.82 & -59.8 & -15.9 \\
Average percentage & +10.1 & -43 & -5 \\
\hline \hline
\end{tabular}

\section{A New Method of $K_{s}$ Allocating; Dividing Technique}

Assigning a single value of $K_{s}$ to the springs beneath the footing leads to a uniform settlement of the whole footing, which is in contrast with given results using more advanced geotechnical softwares. As discussed formerly, system rigidity influences the resulted footing deformation. To further clarify, the settlement of $10 \times 10$ footings with distinct rigidities subjected to a $100 \mathrm{kPa}$ uniform loading and are given in Fig. 3. The flexible and semi-flexible footings show a dish-shaped behavior while others settle uniformly.

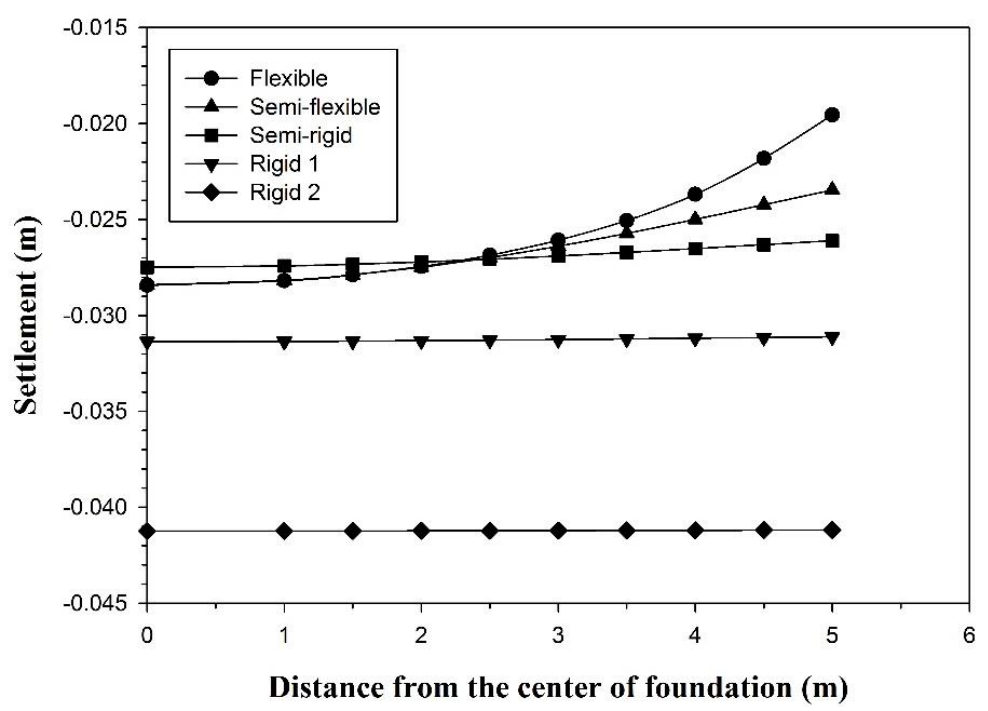

Fig. 3. The effect of rigidity on the foundation's shape after loading.

For semi-rigid and two rigid cases the difference between the maximum and minimum settlement were negligible values of $5,0.8$, and 0.2 percentages, respectively. 


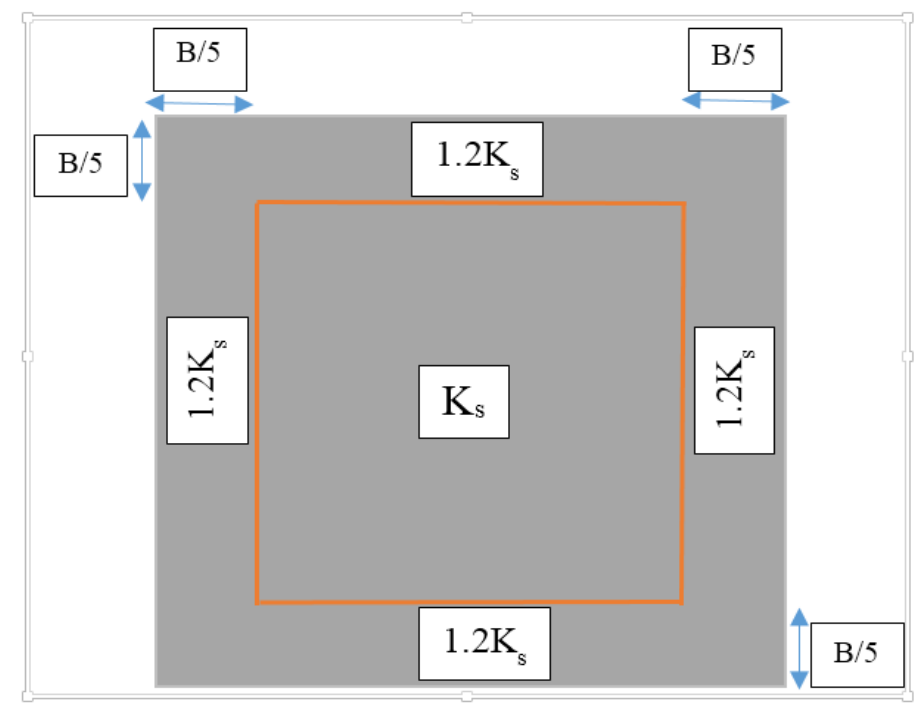

Fig. 4. (Color online) Proposed dividing technique in case of semi-flexible footing.

Thus, the assumption of uniform $K_{s}$ could be adopted in these cases. On the other hand, the discrepancy percentage between maximum and minimum settlements of flexible and semi-flexible footings, i.e. $40 \%$ and $20 \%$, represent the necessity of $K_{s}$ redistribution. The identical discrepancies, i.e. $40 \%$ and $20 \%$, were also observed in $20 \times 20,30 \times 30,40 \times 40$, as well as $50 \times 50$ with the tolerance of $\pm 2 \%$, which reveals that this discrepancy is independent of foundation size and is utilizable generally.

For this purpose, the footing is divided into the central part and side parts with the width of $B / 5, B$ is the foundation breadth, for sides. The $K_{s}$ obtained from empirical relations earmarked to the central part and 1.4 and 1.2 of that were given to the side parts. The proposed dividing technique for semi-flexible case is schematically illustrated in Fig. 4.

For further elucidation and technique verification, $10 \times 10$ footings with different rigidities were analyzed by means of proposed dividing method, using SAFE and compared to the PLAXIS 3D Foundation results. The values of $K_{s}$ were obtained from Biot and elastic-based relations. The results for flexible and semi-flexible footings are presented in Fig. 5 and Fig. 6, respectively. In addition to the settlement comparison, a comparative analysis of the normalized bending moments for different solution is shown, for semi-flexible footing, in Fig. 7. Nevertheless, for the flexible case, the bending moments are inconsequential values close to zero (e.g. $3 \mathrm{kPa}$ at the peak value) that is considered as null bending. 


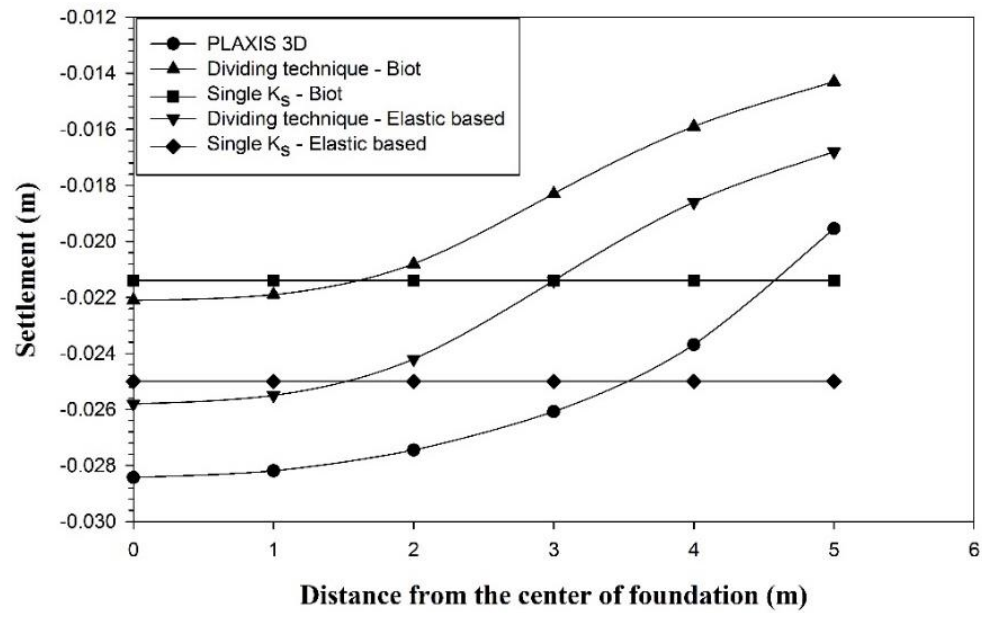

Fig. 5. Comparison among the 3D finite element modeling, proposed dividing technique, and conventional modeling (single $K_{s}$ assignment) results- flexible footing.

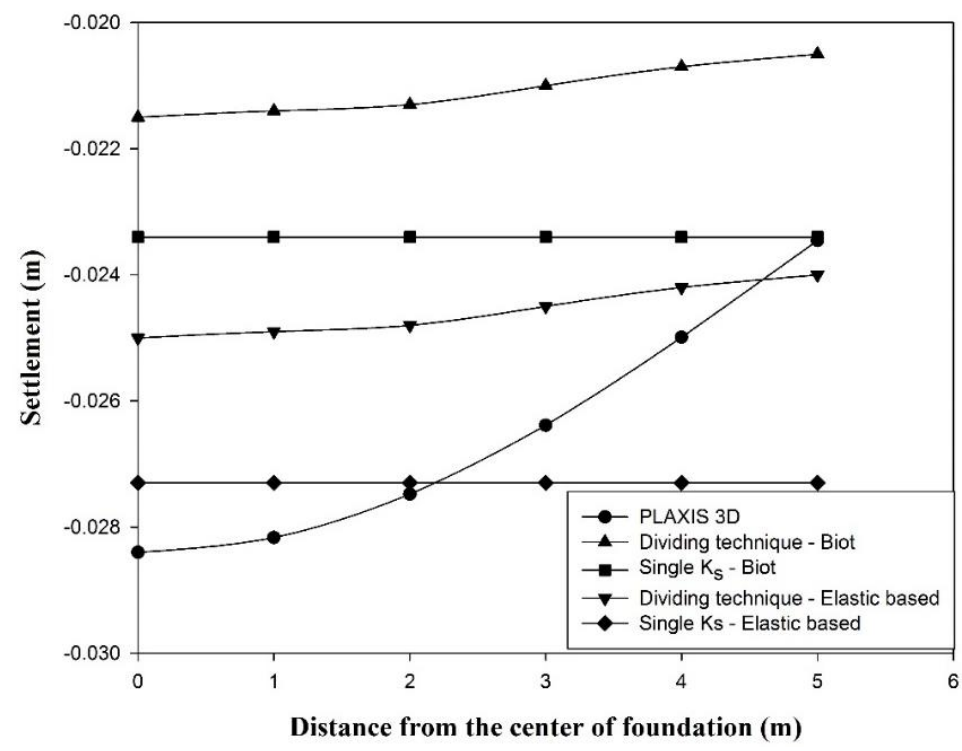

Fig. 6. Comparison among the 3D finite element modeling, proposed dividing technique, and conventional modeling (single $K_{s}$ assignment) results- semi-flexible footing.

In order to make a comparison, the results using conventional modeling technique (assigning a single $K_{s}$ to the footing entirely) is also included into the presented results. 


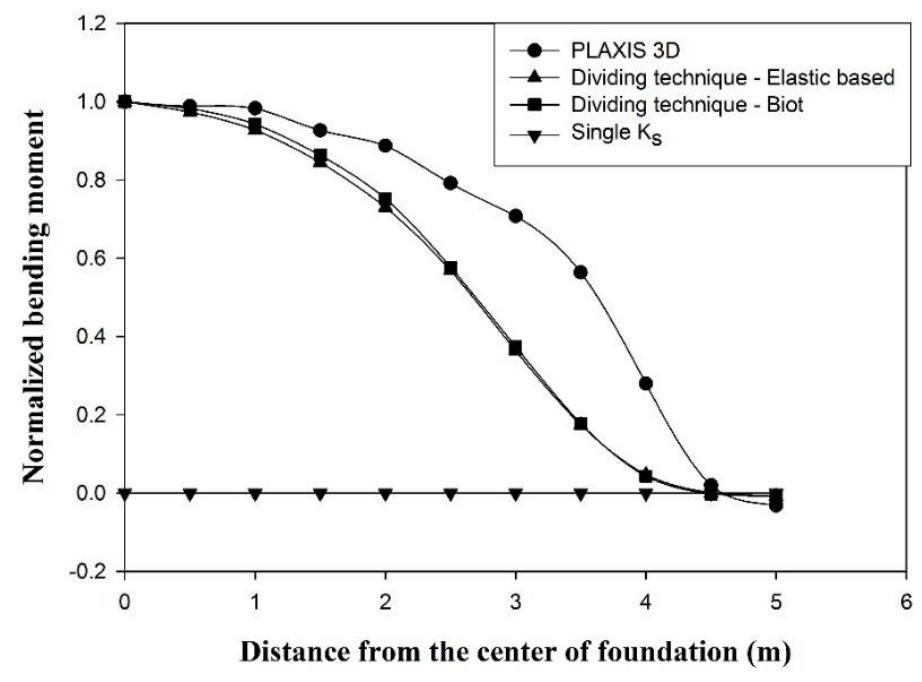

Fig. 7. Bending moments comparison among the 3D finite element modeling, proposed dividing technique, and conventional modeling (single $K_{s}$ assignment) results- semi-flexible footing.

The settlement profile of the footing that used the dividing technique and $K_{s}$ computed by the relation based on theory of elasticity provides the best agreement with the 3D finite element results with the maximum and average deviations of $22 \%$ and $14 \%$, for flexible footing and $12 \%$ and $7 \%$ for semi-flexible footing. However, this deviation using Biot relation and dividing technique are $33 \%$ and $26 \%$ for flexible footing and $24 \%$ and $20 \%$ for semi-flexible footing. The conventional modeling technique is also rendered a uniform settlement profile in both cases. In terms of bending moment, both the Biot and elastic-based relations offer fitted results in comparison with the 3D finite element results, whereas the conventional modelling technique leads to no bending moment generation.

\section{Conclusion}

This research is conducted in response to the gap available in modeling of a foundation using the modulus of subgrade reaction, by introducing a new modeling technique that keeps the simplicity besides the reducing of inevitable errors exist in the Winkler's model. Consequently, a parametric study on the influence of soilfoundation rigidity on the $K_{s}$ distribution of a footing subjected to a uniformly distributed load, is accomplished by using PLAXIS 2D \& 3D softwares. The results indicated that increasing the footing's stiffness resulted in increasing the stress concentration at a distance near the sides of foundation, hence the $K_{s}$ increment. This 
stress concentration is owing to the footing resistance against bending because of its rigidity. Additionally, the semi-rigid footing is utterly as same as the rigid one, in terms of $K_{s}$ distribution.

Comparisons among the $K_{s}$-relation results and finite element code results revealed that the Biot and elastic-based relations have a better estimation of $K_{s}$. Nonetheless, Vesic relation computes the $K_{s}$ with average of $43 \%$ lower than values given by 3D finite element software.

A new method of $K_{s}$ assignment is proposed in this paper. By this method, the foundation surface is divided into the central part and side parts and calculated $K_{s}$ from the experimental and theoretical relations, viz. Biot and elastic-based relations, allocated to the center and increased value, by $40 \%$ and $20 \%$, to the side parts. The best agreement between the finite element and the proposed technique results is obtained by using $K_{s}$ of the relation based on the theory of elasticity. However, the bending moments generated using both Biot and elastic-based relations have an adequate accuracy and agreement with the one obtained from 3D finite element method. Finally, for semi-rigid and rigid cases assigning a single value of $K_{s}$ for entire the foundation is reliable.

\section{REFERENCES}

[1] E. Winkler (1868) “Die Lehre Von Der Elastizität und Festigkrit”. Dominicus, Prag. (in German)

[2] J. Sadrekarimi, M. Akbarzad (2009) Comparative Study of Methods of Determination of Coefficient of Subgrade Reaction. Electronic Journal of Geotechnical Engineering 14(1) 45-61.

[3] H. Farouk, M. Farouk (2014) Calculation of Subgrade Reaction Modulus Considering the Footing-Soil System Rigidity. In: Proceedings of the 2nd International Conference on Vulnerability and Risk Analysis and Management (ICVRAM) and the 6th International Symposium on Uncertainty, Modeling, and Analysis (ISUMA), Liverpool, UK, pp. 2498-2507.

[4] M.A. B Іот (1937) Bending of Infinite Beams on an Elastic Foundation. Transactions of the American Society of Mechanical Engineers 59 A1-A7 [reprinted from Journal of Applied Mechanics, March 1937].

[5] A.B. Vesic (1961) Beams on Elastic Subgrade and Winkler's Hypothesis. In: Proceedings of the 5th International Conference on Soil Mechanic and Foundation Engineering, Paris, pp. 845-850.

[6] J.E. Bowels (1997) "Foundation Analysis and Design", 5th Edition. the McGraw-Hill Co., Illinois.

[7] V.Z. Vlasov, N.N. LeOT'Ev (1966) "Beams, Plates, and Shells on Elastic Foundations". Translated from Russian by the Israel Program for Scientific Translotions, Jerusalem. 
[8] C.V. Vallabhan, Y.C. Das (1988) An Improved Model for Beams on Elastic Foundations. In: Proceedings of the ASME/PVP Conference, Pittsburg, Pennsylvania.

[9] A.T. Daloglu, C.V.G. Vallabhan (2000) Values of K for Slab on Winkler Foundation. Journal of Geotechnical and Geoenvironmental Engineering 126(5) 463-471.

[10] R. Ziaie-Moayed, M. Janbaz (2009) Effective Parameter on Modulus of Subgrade Reaction in Clayey Soil. Journal of Applied Science 9(22) 4006-4012.

[11] A. Arnold, J. Laue, T. Espinosa, S.M. Springman (2010) Centrifuge Modelling of the Behaviour of Flexible Raft. In: Proceedings of the 7th International Conference on Physical Modelling in Geotechnics (ICPMG 2010), Zurich, Switzerland, pp. 679684.

[12] H. FArouk, M. FARouk (2014). Effect of Footing Rigidity on Contact Stress. Presented at Geo-Hubei 2014 International Conference on Sustainable Civil Infrastructure, Yichang, Hubei, China; DOI: 10.1061/9780784478530.010. 